\title{
Correction to: A Review of the Clinical Pharmacology of Pelacarsen: A Lipoprotein(a)-Lowering Agent
}

\author{
Jennifer Hardy ${ }^{1}$. Stephanie Niman ${ }^{1}$ - Rebecca F. Goldfaden ${ }^{1}\left[\right.$ - Majdi Ashchi ${ }^{2} \cdot$ Mohannad Bisharat $^{2}$. \\ Jessica Huston ${ }^{3} \cdot$ Heather Hartmann ${ }^{1} \cdot$ Rushab Choksi ${ }^{1}$
}

@The Author(s), under exclusive licence to Springer Nature Switzerland AG 2021 / Published online: 11 December 2021

\section{Correction to: American Journal of Cardiovascular Drugs https://doi.org/10.1007/s40256-021-00499-1}

In this article Fig. 2 was inadvertently published as Fig. 2a and $2 \mathrm{~b}$

The Fig. 2 should have appeared as shown below.

The original article has been corrected

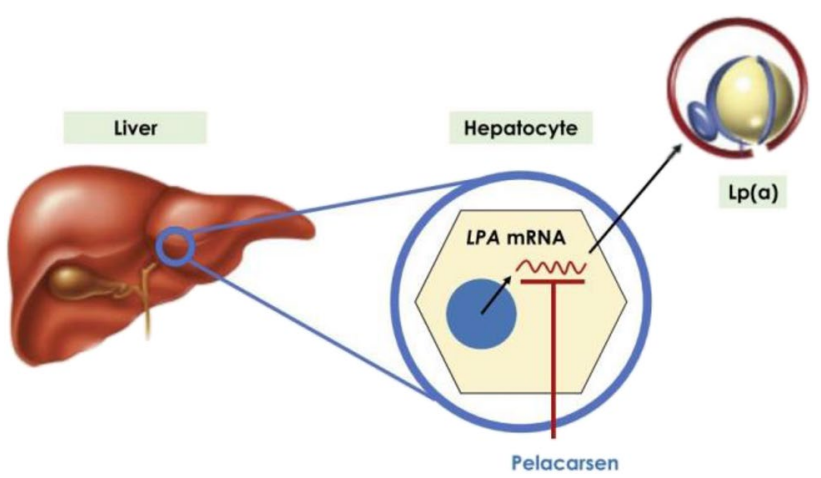

Fig. 2 Mechanism of action of pelacarsen [20, 31]. Schematic overview of pelacarsen's mechanism of action. Pelacarsen targets LPA gene mRNA and prevents apo(a) production leading to decrease Lp(a) particle assembly.

The original article can be found online at https://doi.org/10.1007/ s40256-021-00499-1.

Rebecca F. Goldfaden

Rebecca.Goldfaden@ecirmed.com

East Coast Institute for Research, Jacksonville, FL, USA

2 Ashchi Heart and Vascular Center, Jacksonville, FL, USA

3 College of Pharmacy, University of Florida, Jacksonville, FL, USA 